\title{
Partner Aggression Versus Partner Abuse Terminology: Moving the Field Forward and Resolving Controversies
}

\author{
Robert Geffner ${ }^{1}$
}

Published online: 4 November 2016

(C) Springer Science+Business Media New York 2016

This special issue has three goals: first to recognize Dr. Murray Straus and his legacy in the family violence field; second, to attempt to move the field of family violence forward with new research that focuses on key issues in perpetration of physical partner aggression; and third, to help resolve issues of terminology, definitions, and constructs that have led to controversy and disagreements among those trying to understand and reduce intimate partner violence (IPV) and abuse. Some of the controversies have been occurring for at least 30 years.

I am deeply honored to have been chosen by Drs. Murray Straus and Zeev Winstok to edit this special issue. I knew Murray for over 30 years, and he was the key person who set into motion the elements that has led to my long career and work in this field. In the early 1980's, when I was just beginning to think about starting a family violence institute that would act as a clearinghouse for current information about research, programs, and prevention of family violence, I sent letters to leaders in the field inviting their input, feedback, and participation. At that time, I was an unknown Assistant Professor of Psychology at the University of Texas at Tyler. I did not receive many responses, but one I received within a few weeks was a handwritten letter from Murray encouraging me to move forward, providing some feedback, and offering to help in any way he could. That personal letter enabled me to contact others again, noting we were going to move forward, and indicating that Murray and a few other key people were on our advisory board.

Robert Geffner

bgeffner@alliant.edu

1 Institute on Violence, Abuse \& Trauma, San Diego, CA, USA
That was the beginning of the nonprofit Family Violence \& Sexual Assault Institute that continues today in San Diego, CA. It was also the beginning of a warm, collegial friendship in which Murray and I would exchange articles and ideas, have discussions and sometimes disagreements, and also attend each other's conferences. It was clear when this special issue was initiated, Murray knew he would not be alive to see its completion, but as was his style, that did not deter him. He wanted to make one last attempt to resolve some key issues, and to do it in an innovative manner. This special issue has twice as many articles as is usual for the journal, but each article was planned to be about half the number of pages usually allowed. The response to Murray and Zeev's Call for Manuscripts was amazing. Through the usual peer review process, those submissions have been narrowed to the articles in this special issue. Zeev has done an admirable job of guiding this special issue to its completion, editing articles, and ensuring the high quality of the material. We are all indebted to Zeev for his excellent work and persistence.

Emily Douglas' Introduction in this issue presents the history in brief of Murray's many contributions and life work. I won't repeat them here. However, one of the main issues that Murray and I disagreed about in our past discussions, gender symmetry versus asymmetry in the perpetration of IPV and abuse, has been a source of much disagreement and contentious debate in the field. This disagreement has led to loud and sometimes angry arguments for 30 years by those who are involved in research, policy, advocacy, and/or treatment of intimate partner violence (IPV). Murray and other researchers have been vilified for suggesting that women were as violent as men in relationships (i.e., gender symmetry). Although the debate over gender symmetry continues, there has been no disagreement that in IPV situations, women are much more likely to be injured and also more severely than men. 
What continues to be argued is whether women are as likely to be as violent, aggressive, or abusive in relationships as men. This issue has kept the field from advancing. Some have seen Murray's work as the main problem in that it has been perceived to minimize the effects of abuse on women. The counterpoint is that many have not been willing to recognize that women can be violent and abusive in relationships, and they also do not see women's use of aggression as equivalent to that of male perpetrators with respect to danger, severity, or effect. They therefore reject the concept of gender symmetry in abusive relationships. My discussions with Murray in the last few years have focused on terminology and the issues of gender within a social conceptual framework. Sadly, the choice of words people have been using for a few decades now have exacerbated this debate and polarized people in the field, with little middle ground. Some still argue the more extreme position that women are as, or are even more, abusive than men, stating that this has been ignored because of gender bias against men. Others argue that men are the primary perpetrators of IPV which causes severe trauma for many women worldwide, and such levels of violence and abuse are often minimized due to the patriarchal views of men. Others have focused on measurement issues in dealing with the complexities of studying violence and abuse, and they have viewed the issues of gender within a social ecological perspective. Some family violence researchers have argued that practitioners and advocates have ignored the research data, and advocates and practitioners have argued that the researchers have not viewed abuse and its effects in the larger sociocultural context, or acknowledged the differences between violence by men and by women.

The terminology used in the field of intimate partner violence is still inconsistent, which has contributed to the strong feelings on both sides of these issues. Uniform and coherent terminology is a precondition for effective communication among practitioners, advocates, researchers, and others, especially in light of the field's multiple controversies. People in the field have used such terms as violence, abuse, aggression, and assault as if they are interchangeable in publications, presentations, discussions, legal statutes, and policies. The loose use of terminology has sometimes sidetracked us from looking at the individualistic versus sociocultural perspectives, and alternative explanations that might be more helpful in reconciling the research data within the sociocultural context that does include gender issues. These issues have contributed to the disagreements, and have hindered the field from unifying and progressing. In the last few years, Murray regretted that the interpretation and portrayal of his work, by himself and others, led to such disagreements and strong arguments that hindered advancement of the field.

This special issue of the journal was an attempt to move forward with some new frameworks. I am also suggesting that definitions and the use of such terminology and our language be tightened with respect to how we describe and label relationship violence. There are people who will likely remain at both extremes of the gender symmetry/asymmetry positions, but I am hopeful that the majority of those who want to see the end of IPV will help move the field of family violence forward, and will look for common ground in viewing violence and abuse in relationships.

The first step is the definitions of terms. I am not going to focus on legal definitions because they vary too much and do not help us move forward. Assault and violence are usually physical and/or sexual acts and behaviors of aggression, but they can also be psychological, though we do not often think of psychological assault. These terms refer to one person striking out physically, sexually, or psychologically at another. For IPV, it occurs in a relationship. Abuse not only includes the above, but adds a few other key components. Whereas the terms above usually refer to isolated events, abuse generally involves an ongoing pattern that may include multiple forms of aggression. Abuse assumes there is a relationship between the participants, and also takes into account the intent as well as the consequences on the person being victimized. For example, abuse has been characterized by the use of power and coercive control where one person uses his/her superior position, privilege, or strength to impose their will on another, usually through intimidation. The control may be directed at the victim's actions, feelings, and/or beliefs. Abuse produces fear, anxiety, helplessness, and trauma which can have further adverse psychological and physiological consequences, including post-traumatic stress disorder. Thus, the term abuse also considers context, motivation, and consequences, which then invokes the sociocultural framework and gender issues that are crucial to better understanding the dynamics of these situations.

When these definitions are used, it becomes clear that abuse and aggression/violence are not the same, even though they have overlapping features. Thus, there can be abuse in relationships without physical violence/aggression, there can be violence/aggression in relationships without abuse (i.e., without power, control, intimidation, trauma), or abuse and violence/aggression together in relationships. It is important to distinguish these terms and the overall framework because they are keys to the field progressing. It is also important to keep in mind the consequences of the actions, as well as the frequency, the duration, and the severity of the behaviors when we discuss these situations or attempt to measure these behaviors. Considering the behaviors alone, or even the outcome, do not provide the full picture of motivation, intent, or the psychological effects and dynamics. With such definitions, mutual abuse would be less likely, though still possible (e.g., both partners striving to use violence/aggression to intimidate as well as to control the other person). On the other hand, mutually aggressive behaviors might be much more likely in the context of arguments or disagreements in a relationship. 
We need to be more precise in what we are referring to, whether it regards research, practice, advocacy, or policy.

Thus, from decades of research that Murray Straus and many others have conducted, it is clear that both men and women may be aggressive in relationships. However, it is also clear that much of the aggression in relationships, especially female-to-male aggression, does not meet the definition of abuse noted above due to the lack of an ongoing pattern of coercive control and the intent to dominate and intimidate. When the above definitions are used, abusers are primarily men. Women are much more likely to be the recipients of the most trauma, fear, being controlled, and serious injuries in such relationships. Characterizing intimate partner abuse as gender symmetrical is simply not accurate then.

Similarly, denying that women use aggression in relationships does not match the research data. In fact, women may use similar forms of aggression as men, cause injury, and behave violently, but they would not necessarily be acting abusively according to the definition above. Further, regardless of definitions, violence and abuse by either partner in an intimate relationship is unacceptable, and we need to keep working to eliminate it. This is more likely to happen if those working to end violence and abuse can embrace their common goal and stop losing time and energy fighting with each other. Murray cared deeply about ending violence and abuse in the family. It was a source of consternation to him that wellmeaning people with the same goals spent so much time attacking each other. It would be a fitting legacy to Murray if this special issue helps us to move forward together to end relationship violence and abuse.

Acknowledgments I would like to express my appreciation to many colleagues and friends for providing valuable feedback on this introduction, and for their important work to ending interpersonal violence and abuse (Alan Rosenbaum, Jacquelyn White, Glenna Tinney, Zeev Winstok, and Sandi Capuano Morrison). However, this is not meant to imply that the views expressed in this introduction are those of these other colleagues. 